\title{
Validación de una escala de afrontamiento frente a riesgos extremos
}

\author{
Esperanza López-Vázquez, D ra en Psicol Soc,(1) María Luisa Marván, Dra en C.. (1)
}

\section{López-Vázquez E, Marván ML. Validación de una escala de afrontamiento frente a riesgos extremos.} Salud Publica Mex 2004;46:216-221. El texto completo en inglés de este artículo está disponible en: http://www.insp.mx/salud/index.html

\section{Resumen}

Objetivo. Validar, en población mexicana, una escala de afrontamiento, adaptada de la escala francesa "Echèlle Toulousaine de Coping". Material y métodos En el otoño de 2001 la escala se aplicó a 209 sujetos que habitaban en diversas zonas de México, expuestos a cinco diferentes tipos de riesgo extremo, entre los cuales se distinguen riesgos naturales y riesgos industriales. Se analizó la capacidad discriminatoria de los reactivos, así como la estructura factorial y la consistencia interna de la prueba. Se emplearon los métodos U de Mann-W hitney, análisis factorial de componentes principales y alpha de Cronbach. Resultados La escala final es de 26 reactivos que se agruparon en dos factores: afrontamiento activo y afrontamiento pasivo. La consistencia interna del instrumento es muy alta, tanto en la muestra total como en la submuestra de riesgos naturales y riesgos industriales. Conclusiones La escala de afrontamiento que proponemos es confiable y válida para la población mexicana. El texto completo en inglés de este artículo está disponible en: http://www.insp.mx/salud/index.html

Palabras clave: afrontamiento; riesgos extremos; riesgos naturales; riesgos industriales; México

\author{
López-Vázquez E, Marván ML. \\ Validation of a scale measuring \\ coping with extreme risks. \\ Salud Publica Mex 2004;46:216-221. \\ The English version of this paper \\ is available at: http://www.insp.mx/salud/index.html
}

\begin{abstract}
A bstract
Objective. The objective of this study was to validate, in Mexico, the French coping scale "Échelle Toulousaine de Coping". Material and Methods In the fall of 2001, the scale questionnaire was applied to 209 subjects living in different areas of Mexico, exposed to five different types of extreme natural or industrial risks. The discriminatory capacity of the items, as well as the factorial structure and internal consistency of the scale, were analyzed using MannW hitney's U test, principal components factorial analysis, and Cronbach's alpha. ResultsThe final scale was composed of 26 items forming two groups: active coping and passive coping. Internal consistency of the instrument was high, both in the total sample and in the subsample of natural and industrial risks. Conclusions. The coping scale is reliable and valid for the Mexican population. The English version of this paper is available at:http://www.insp.mx/salud/index.html
\end{abstract}

Key words: coping; extreme risks; natural risks; industrial risks; Mexico
E 1 riesgo de un evento extremo, cuyas consecuenE cias pueden ser catastróficas, es una condición con la cual el ser humano ha vivido a lo largo de su historia. Las experiencias relacionadas con las catástrofes naturales durante mucho tiempo fueron consideradas como un castigo divino al cual no se podía el hombre oponer. Luchando contra esta contrariedad, el hombre implementó los medios para poder defenderse y

(1) Departamento de Psicología de la Universidad de las Américas-Puebla, Puebla, México.

Fecha de recibido: 17 de enero de 2003 - Fecha de aprobado: 20 de febrero de 2004 Solicitud de sobretiros: D ra. Esperanza López Vázquez. D epartamento de Psicología, Universidad de las Américas-Puebla, Ex Hacienda Santa Catarina Mártir s/n, 72820 Cholula, Puebla, México. Correo electrónico: espelv@ mail.udlap.mx 
retornar la naturaleza contra ella misma. Sin embargo, los mismos medios que creó con el fin de defenderse, han terminado por revelar una nueva amenaza que es el riesgo tecnológico mayor, contra el cual también ahora debe defenderse. ${ }^{1}$ Actualmente, se puede decir que casi todos los peligros o amenazas de la naturaleza presentan elementos tanto tecnológicos como naturales. ${ }^{2}$ Durante el siglo $X X$, se calcula que alrededor de tres billones de personas fueron afectadas por algún desastre (miles fueron afectadas sin duda en múltiples ocasiones en este periodo). La experiencia de tales eventos ha dejado consecuencias psicológicas ligadas a dichos desastres que, en múltiples casos, han alterado la salud de los individuos. ${ }^{*}$ Dichas alteraciones que se conjugan en una serie de síntomas, denominados síndrome de estrés postraumático (PTSD, por sus siglas en inglés), se observan con mayor intensidad, tanto en víctimas de catástrofes colectivas como en situaciones de estrés individual. ${ }^{3}$ Una evaluación psicológica hecha a las 10 semanas del terremoto de México en 1985, mostró que $32 \%$ de los damnificados presentaba PTSD. ${ }^{4}$ En las últimas décadas, tanto el gobierno como los científicos y la población en general han mostrado un creciente interés por manejar y prevenir las amenazas a la salud y el bienestar humano. ${ }^{5}$

La forma como enfrentamos el peligro va a depender de muchos factores que se pueden ver resumidos en la evaluación cognitiva que los individuos realizan frente a un evento. Todos los esfuerzos cognitivos y de comportamiento, que cambian constantemente y que se utilizan para gestionar las demandas específicas, tanto externas como internas, que se evalúan como excediendo los propios recursos, se conocen como estrategias de afrontamiento. ${ }^{6}$ Algunos estudios realizados en México han mostrado que la gente expuesta, ya sea a un riesgo sísmico o industrial, tiende a utilizar más estrategias pasivas que activas. ${ }^{7}$ Otros estudios muestran que la población mexicana que vivió el terremoto de 1985, donde hubo fuertes pérdidas tanto humanas como materiales, tiende a no hacer nada frente al riesgo sísmico, aceptando las pérdidas en caso de que un evento de este tipo se repita. ${ }^{8}$ Otros estudios han demostrado también cómo las culturas individualistas tienden a reforzar los sesgos positivistas, la falsa unicidad, sentimiento de invulnerabilidad y el optimismo ilusorio, a diferencia de las culturas colectivistas, quienes reaccionan más en conjunto y menos de manera individual. ${ }^{9}$

\footnotetext{
* Fernández I, Beristáin C, Páez D. Emociones y conductas colectivas en catástrofes: ansiedad y rumor, miedo y conductas de pánico.1998. Manuscrito no publicado.
}

Para estudiar el comportamiento de los individuos frente a situaciones estresantes, se han creado numerosas escalas que pretenden medir los diferentes estilos y estrategias de afrontamiento. Dichas escalas se han elaborado en otros países y no se han validado en México. Con el fin de entender mejor los estilos de estrategias de afrontamiento de la población mexicana frente a riesgos extremos es necesario estudiar los comportamientos de los sujetos sometidos a tales riesgos, a través de una escala confiable. Así, el objetivo de este trabajo fue validar en México una escala de afrontamiento inspirada en la escala francesa "Échelle Toulousaine de Coping" en su primera versión de 1992 (anexo I). ${ }^{10} \mathrm{La}$ importancia de tener una escala de esta naturaleza estriba en que puede permitirnos conocer mejor el comportamiento de las personas que viven bajo el constante azote de riesgos, y así poder pensar mejor en una posible intervención. Este tipo de escalas pueden entonces reproducirse en diferentes situaciones de riesgo.

\section{Material y métodos}

\section{Sujetos}

Se estudiaron 209 sujetos expuestos a diferentes riesgos extremos: 99 a riesgos naturales y 110 a riesgos industriales. Se encuestaron 107 hombres y 102 mujeres. Hubo 121 sujetos que tenían entre 17 y 30 años de edad, y los 88 restantes tenían más de 31 años de edad. En general, los sujetos presentaban bajos niveles de escolaridad, muchos de ellos con primaria o secundaria, ya sea incompleta o terminada. En cuanto a los riesgos naturales, 60 sujetos eran habitantes de las zonas cercanas al volcán Popocatepetl (éste se encuentra actualmente en actividad), 22 sujetos de una zona de inundaciones en la Sierra de Puebla, y 21 de una zona sísmica de la Ciudad de México (Tlatelolco). En lo referente a los riesgos industriales, 50 sujetos eran habitantes de la zona industrial de San Juan Ixhuatepec donde se manejan petróleo, diferentes productos químicos y tóxicos en la parte norte de la Ciudad de México, y otros 50 sujetos habitaban la zona cercana a la central nuclear de Laguna Verde, en el estado de Veracruz. La forma como se seleccionó a los participantes fue por disponibilidad, es decir, se les contactó en las calles, comercios o en sus casas. El único requisito particular que se buscó cubrir fue el que tuvieran por lo menos cinco años viviendo en la zona de riesgo.

\section{Instrumentos}

Escala de estrategias de afrontamiento adaptada de la "Échelle Toulousaine de Coping", con 36 preguntas que 
se contestan en una escala Likert de cinco puntos: a) para nada, b) rara vez, c) a veces, d) seguido, y e) muy seguido.

\section{Procedimiento}

El cuestionario original constaba de seis dimensiones teóricas (focalización, soporte social, retraimiento, conversión, control y rechazo) y tres campos (acción, información y emoción). A cada campo corresponden tres reactivos de cada dimensión, en total 54 reactivos. Las dimensiones teóricas fueron establecidas por el laboratorio a cargo, a partir de un análisis crítico de otras escalas de afrontamiento existentes. ${ }^{10}$ Los criterios específicos utilizados para determinar dichas dimensiones se desconocen, pero corresponden a una síntesis de la literatura revisada por ellos. Para nuestro estudio se decidió reducir la extensión de la escala, puesto que tomaba mucho tiempo para contestarla, y se conservaron así dos reactivos de cada campo ligados a cada una de las dimensiones propuestas, quedando así 36 reactivos. Debido al interés por conocer un poco más sobre la estrategia de control relacionada con la internalidad-externalidad de los sujetos, se agregaron dos reactivos más relacionados con este concepto que fueron: "cualquier cosa que pase depende de mí", y "esta situación es debida a la mala suerte". Esto último sólo con la idea de tener un indicador del concepto y no como una medida estricta. El cuestionario final de 38 reactivos fue traducido al español. Una vez concluida la traducción, se hicieron algunas modificaciones en la redacción de algunos reactivos, de tal manera que se adecuaran más a las situaciones de riesgo. Se aplicó a los participantes, respetando la escala Likert original. Así, a cada reactivo se le otorgó una calificación de 1 a 5 .

Las aplicaciones se realizaron en el otoño de 2001. Debido a la dificultad de algunas personas para contestar la escala correctamente, el encuestador participó con ellos en la mayoría de los casos, es decir, éste leía en voz alta cada reactivo y, después de asegurarse de que el sujeto lo había entendido, anotaba en la hoja la respuesta que le daba. Esta situación se presentó debido a que la mayoría de las personas pedían ellas mismas ser ayudadas por el encuestador. Sólo algunas personas, como fue el caso de estudiantes de bachillerato, aceptaban contestarla por ellos mismos (10\% aproximadamente). Pensamos que las posibles repercusiones en cuanto a la forma de llenado deben ser mínimas debido a que el porcentaje de quienes lo contestaron solos es muy bajo, que las explicaciones eran claras y que el encuestador estaba siempre cerca para resolver cualquier duda.

\section{A nálisis de datos}

Se realizó un análisis de la capacidad discriminatoria de los reactivos, el cual se refiere al grado en el que cada reactivo distingue entre los sujetos con mayores y menores puntuaciones totales del instrumento. Se utilizó el método de los grupos extremos, contrastando las medias de los grupos formados por $25 \%$ de los sujetos con puntajes más elevados y $25 \%$ de los sujetos con puntajes más bajos. ${ }^{11}$ Para este análisis se utilizó la prueba U de Mann-Whitney $(p<0.01)$. No se encontraron diferencias significativas en cuatro reactivos, por lo que éstos fueron eliminados.

Posteriormente, se utilizó el método de componentes principales con una rotación varimax ortogonal, para establecer la estructura factorial del cuestionario. ${ }^{12}$ Debido a que este análisis manifestó la presencia de nueve factores con valores propios mayores de uno, se consultó el gráfico de guijarros. Este gráfico representa la magnitud de cada valor propio como una función de su posición serial, lo que permite identificar el número apropiado de factores que se deben extraer. ${ }^{12}$

Para la asignación de los reactivos a cada factor se utilizaron dos criterios: a) que el reactivo estuviera conceptualmente relacionado con el factor considerado, y b) que tuviera un peso factorial mayor a 0.35 en el factor correspondiente.

Finalmente, para determinar la confiabilidad del cuestionario se obtuvo la consistencia interna de cada uno de los factores, así como de la escala general, utilizando el alpha de Cronbach.

\section{Resultados}

El análisis de componentes principales arrojó nueve factores con valores propios superiores a 1 . Sin embargo, al consultar el gráfico de guijarros se observaron únicamente dos factores bien definidos. Por lo tanto, se rehizo el análisis considerando únicamente dos factores que explican $31.65 \%$ de la varianza total. A pesar de que el porcentaje de la varianza es bajo, es un valor aceptado en los estudios realizados dentro de las ciencias sociales, debido a que para explicar cualquier fenómeno social intervienen múltiples variables.

El primer factor agrupó 14 reactivos que explican $17.71 \%$ de la varianza. Estos reactivos se refieren a comportamientos de acción directa sobre el problema, búsqueda de información, estrategias de anticipación a un desastre, control de sí mismo y de las circunstancias $y$, finalmente, de soporte social, por lo que se le denominó afrontamiento activo. El segundo factor quedó compuesto por 12 reactivos que explican $13.93 \%$ de la varianza. Dichos reactivos se refieren a comportamien- 
tos de rechazo y negación del evento, retraimiento y aceptación pasiva, por lo que se le denominó afrontamiento pasivo. Finalmente, hubo ocho reactivos que se eliminaron porque obtuvieron un peso factorial menor de 0.35 en los dos factores.

El índice de consistencia interna del cuestionario general, utilizando el alpha de Cronbach, es de 0.81. El cuadro I muestra la consistencia interna de los factores de afrontamiento activo y pasivo, en las submuestras específicas de sujetos que están sometidos a desastres naturales o desastres industriales. Se puede observar una alta confiabilidad en todos los casos.

En el cuadro II se presentan los 26 reactivos de la variante final del cuestionario, así como sus pesos factoriales.

\section{Discusión}

Los resultados obtenidos permiten presentar un cuestionario válido y confiable, dirigido a la población mexicana, que mide las estrategias de afrontamiento de los sujetos que viven en situaciones de riesgo natural o industrial. A diferencia de la escala validada en Francia, que consta de seis dimensiones teóricas (focalización, control, soporte social, conversión, retraimiento y rechazo), nuestra escala presenta únicamente dos factores: afrontamiento activo y afrontamiento pasivo. Esto parece ser un indicador de diferencias culturales importantes, pero no descartamos otros elementos de base. Es importante señalar que el cuestionario diseñado en Francia se aplicó a estudiantes de preparatoria, universidad y trabajadores sociales, sobre situaciones estresantes anteriormente vividas que los entrevistados mismos escogían para resolver el cuestionario. Es evidente que la diferencia situacional es un elemento que puede explicar en parte las diferencias aquí encontradas. El estrés provocado por una situación extrema no es el mismo que el de una situación banal, dadas las características del elemento extremo, los sujetos generan reacciones diferentes..$^{13}$ Así, los mecanismos de defensa y los comportamientos habituales no son los mismos en ambos casos. En el caso del presente cuestionario, los sujetos entrevistados eran personas expuestas a riesgos extremos. De hecho, $73 \%$ de la muestra había experimentado de alguna manera una catástrofe o bien una situación de riesgo extremo. Las personas expuestas al riesgo volcánico habían sido evacuadas; las expuestas al riesgo industrial habían vivido la explosión de la gasera en San Juan Ixhuatepec en 1984; las expuestas al riesgo sísmico habían vivido un terremoto en 1985; y las expuestas a inundaciones habían vivido algún deslave. Solamente en Laguna Verde nadie había vivido un desastre. Este hecho

\section{Cuadro I \\ Consistencia interna del CUestionario POR FACTORES Y TIPO DE RIESGO}

\begin{tabular}{lll} 
Tipo de riesgo & Afrontamiento activo & Afrontamiento pasivo \\
Riesgo industrial & $\alpha=0.85$ & $\alpha=0.77$ \\
\hline Riesgo natural & $\alpha=0.79$ & $\alpha=0.76$ \\
\hline Total & $\alpha=0.83$ & $\alpha=0.77$
\end{tabular}

\section{Cuadro II \\ Matriz factorial rotada de la escala de AFRONTAMIENTO}

Factor 1 Factor 2

\begin{tabular}{lll} 
Tengo un plan preventivo y lo sigo & 0.656 & \\
\hline Me fijo objetivos y redoblo esfuerzos & 0.636 & \\
\hline Analizo las circunstancias para saber qué hacer & 0.628 & \\
\hline Reflexiono sobre las estrategias a utilizar & 0.619 & \\
\hline He establecido mi propio plan de prevención & & \\
y lo pongo en marcha & 0.630 & \\
\hline Hago modificaciones en mi entorno para evitar & & \\
un desastre & 0.619 & \\
\hline Busco información con per sonas que saben & 0.597 & \\
\hline Trato de no precipitarme y de reflexionar sobre & & \\
los pasos a seguir & 0.573 & \\
\hline Participo más en actividades de prevención civil & 0.550 & \\
\hline Hablo con mi familia para compartir emociones & 0.534 & \\
\hline Consulto sobre el problema con profesionales & 0.471 & \\
\hline Trato de cambiar mis hábitos de vida en función & & \\
del problema & 0.425 & \\
\hline Hago frente directamente a la situación & 0.410 & \\
\hline Controlo en todo momento mis emociones & 0.382 & \\
\hline Trato de no pensar en el problema & & 0.73 \\
\hline Trato de no sentir nada & & 0.678 \\
\hline Hago como si el peligro no existiera & & 0.617 \\
\hline Me paseo para distraerme & & 0.589 \\
\hline Rechazo la idea que esta situación es grave & & 0.575 \\
\hline Deseo un milagro y ruego a Dios para que me ayude & & 0.554 \\
\hline Busco actividades para pensar en otra cosa & & 0.444 \\
\hline Me es difícil describir lo que siento frente & & \\
\hline a esta situación & & 0.424 \\
\hline Bromeo y tomo las cosas a la ligera & & \\
\hline Sigo lo que hacen los demás & & \\
\hline Algunas veces no hago lo que ya había previsto hacer & & 0.394 \\
\hline Acepto la situación pues es inevitable & & \\
\hline
\end{tabular}




\section{Anexo I}

\section{Escala de AFrontamiento}

Le presentamos a continuación una lista de afirmaciones. Usted debe indicar con qué frecuencia se identifica con dichas afirmaciones. Especifique su respuesta poniendo una cruz $(\mathrm{X})$ en el cuadro que corresponda con la opción con la que usted más se identifique. No hay respuestas correctas 0 incorrectas. Gracias

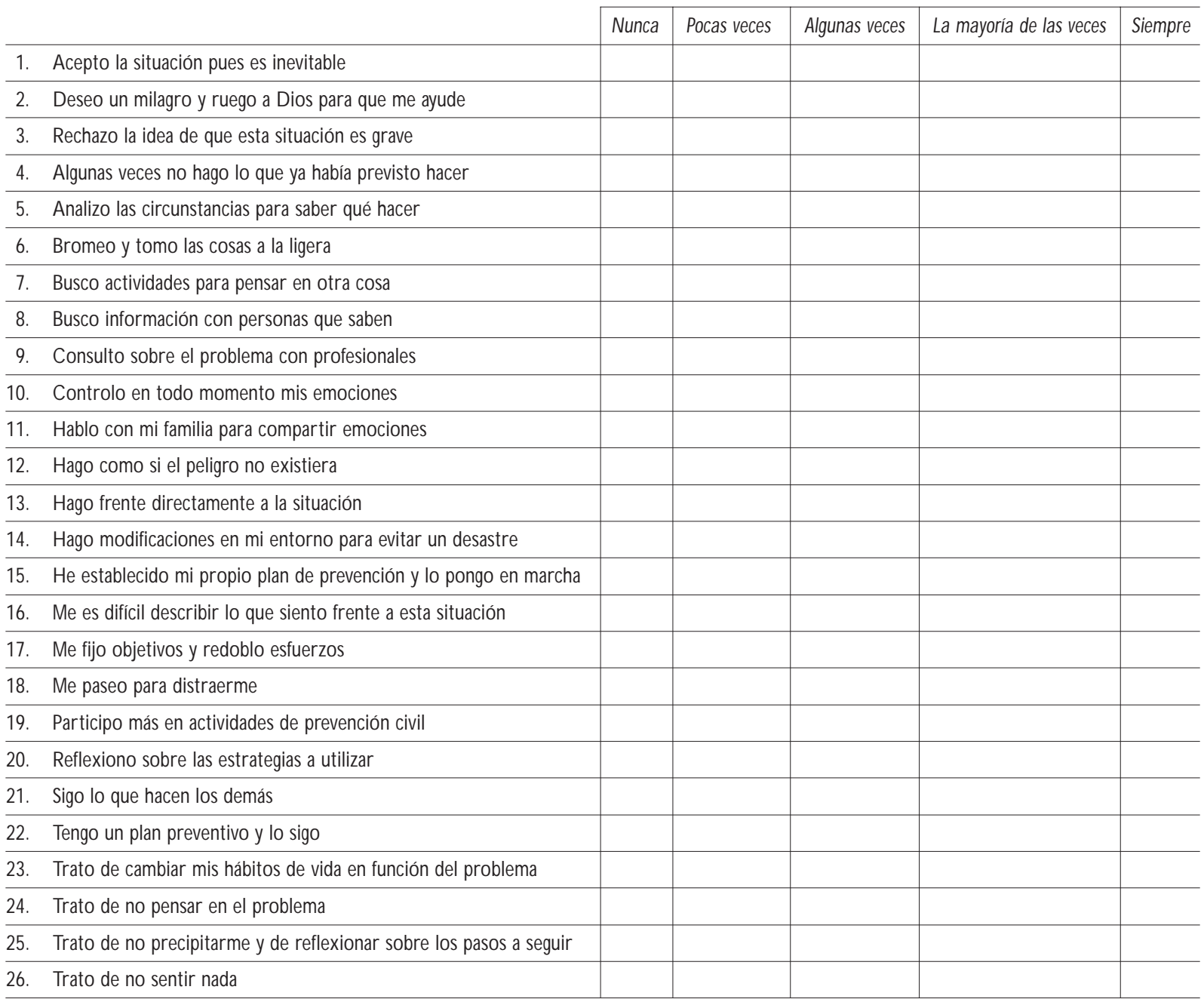

puede considerarse como una posible limitación de la presente investigación, ya que la intención no es que se entreviste únicamente a personas con la experiencia previa de un desastre. En estudios futuros se deberán comparar los resultados de esta escala en gente que ha vivido algún desastre y gente que no ha tenido la experiencia. La experiencia previa sin duda afecta la percepción que se tiene del peligro, y por lo tanto repercute en la forma como lo afrontan. En el caso de las personas que viven cerca de la central nuclear, aun cuando no han vivido un desastre, han percibido por diferentes medios de comunicación las consecuencias de un posible desastre. Así, concluimos que para todos los sujetos de alguna forma está claro que la situación sobre la cual fueron interrogados es una situación extrema. Por otro lado, nuestro cuestionario es útil para medir el afrontamiento, tanto en una situación de riesgo natural como industrial, tal y como lo indica el índice de confiabilidad. Esto puede ser debido a que el peligro percibido y los recursos que se pueden utilizar 
frente a una u otra situación remiten a procesos psicológicos similares.

Es importante señalar que las diferencias culturales pueden ser un factor decisivo en las diferentes formas de afrontar una situación estresante. Un estudio hecho en Guatemala por la Oficina de Derechos Humanos del arzobispado de Guatemala en 1998, sobre el afrontamiento en situaciones de catástrofe humanitaria, reveló cinco dimensiones de afrontamiento: afrontamiento directo y auto-control, afrontamiento colectivo de huida colectiva, afrontamiento instrumental colectivo, afrontamiento emocional adaptativo, y afrontamiento cognitivo e ideológico. ${ }^{9}$ Otros estudios hechos en México sobre los estilos de afrontamiento de los mexicanos revelaron la existencia de tres estilos básicos: el estilo directo revalorativo, el estilo emocional negativo y el estivo evasivo. ${ }^{14}$ Otros estudios sobre los tipos de mexicanos han mostrado que el más común en la sociedad mexicana es el del mexicano pasivo y obediente-afiliativo, que se encuentra en las zonas rurales y provincias del centro y sur de la República. ${ }^{15}$ Estos últimos resultados podrían explicar el hecho de que en el presente cuestionario se obtuviera un número de factores diferentes con conceptos diferentes, en este caso únicamente dos factores centrados en estrategias pasivas y en estrategias activas. Se concluye con esto que el tipo de estrategias utilizadas son culturales, y por ello es necesario realizar estudios transculturales para comparar las diferencias en el tipo de afrontamiento utilizado en otras culturas frente a riesgos extremos.

Respecto a la posible utilidad de este cuestionario, podría serlo para la investigación en el área de la psicología social, de la psicología de la salud y de la salud pública. Puede utilizarse como instrumento de medición de reconocimiento de estilos de afrontamiento frente a cualquier tipo de peligro, y de esta forma, poder elaborar programas de prevención o de intervención comunitaria. También puede servir para hacer comparaciones culturales en el seno de la misma cultura o con otras diferentes. Otra posibilidad sería la de evaluar la existencia de cambios en los estilos de afrontamiento antes y después de una intervención determinada o de una catástrofe. Este cuestionario también puede utilizarse para estudiar la relación de las estrategias de afrontamiento con otras variables como el estrés y la percepción del riesgo, ${ }^{16,17}$ el impacto de alguna información de prevención en el aprendizaje social, la influencia de las representaciones sociales; también, con algunas adaptaciones a los ítems puede verse el tipo de afrontamiento frente a enfermedades como el cáncer y la diabetes ${ }^{17,18}$ y otras como el SIDA $y$, en fin, otras variables que contribuyan a entender las reacciones de las personas expuestas a riesgos extremos y también de aquellas que han vivido un desastre, como sería el caso del estrés postraumático.

\section{Agradecimientos}

Las autoras agradecen a Alberto Jiménez Marín por la aplicación de las encuestas y la captura de datos para su análisis.

\section{Referencias}

1. Lagadec P. La civilisation du risque. C atastrophes technologiques et responsabilité sociale. Paris: Seuil; 1994.

2. Martínez-Trovisco J, Hernández B, San Luis C. La percepción de las amenazas naturales. Rev Psicol Soc A pl 1997;7(1-2):15-34.

3. Beristain M, Georgia C, Páez D, Pérez P, Fernández I. Reconstruir el tejido social. Un enfoque crítico de la ayuda humanitaria. Barcelona: Icaria;1999.

4. MarsellaAJ, Friedman MJ, Gerrity ET, Scurfield RM. Ethnocultural aspects of post-traumatic stress disorder. W ashington, DC:A merican Psychological Association; 1997.

5. Cohen R. Salud mental para víctimas de desastres. México, DF: El Manual Moderno; 1998.

6. Lazarus R, Folkman S. Stress appraisal and coping. N ueva York (N Y): Springer Pub; 1984.

7. López-Vázquez E, Marván ML. Risk perception, stress and coping strategies in two catastrophe risk situations. Soc Behav Personal 2003;31(1):61-70.

8. Urbina-Soria J. Percepción de riesgos ambientales en la ciudad más grande del mundo. En:Actas del $1^{\text {er }}$ Seminario $\mathrm{N}$ acional de la Red Mexicana de Estudios Interdisciplinarios para la Prevención de Desastres; 1955 sept; México, DF, México.

9. Páez D, Fernández I, Beristain CM. C atástrofes, traumas y conductas colectivas: procesos y efectos culturales. En: San Juan C, comp. Catástrofes y ayuda de emergencia. Barcelona: Icaria; 2001: 85-148. 10. Esparbes S, Sordes-A der F,Tap P. Presentation del échelle de coping. Les stratégies de coping. Journées du labo 1994 PCS 93. st criq: 89-107. 11. Morales P. Medición de actitudes en psicología y educación: construcción de escalas y problemas metodológicos. San Sebastián, España:Ttarttalo; 1987.

12. N unnally JC, Bernstein IJ. Teoría psicométrica. México, D F: Mac Graw-Hill; 1995.

13. N oto R. Stress et catastrophe. N euro-psy 1989; 4 (4): 201-206. 14. Reyes-Lagunes I. Conferencia en elV Congreso de Psicología en la Universidad de las A méricas, 2002 nov 6-9; Puebla, México.

15. Díaz-Guerrero R. Psicología del mexicano. Descubrimiento de la etnopsicología. 6a edición. México, DF:Trillas; 1994.

16. López-Vázquez E. Risk perception interactions in stress and coping facing extrem risks. Environ Manag Health 2001;12(2,3):122-133.

17. Esparbes-Pistre S.Anxiété et stratégies de faire face chez des adolescents gravement malades (cancer, diabète). C ah Int Psych Soc 1997;33:109-121.

18. Sordes-Ader F. Les stratégies de coping chez les adolescents atteints de cancer. Cah Int Psych Soc 1997;33: 97-107. 